\title{
Double Ionization of Helium by Electron-Impact: Complete Pictures of the Four-Body Breakup Dynamics
}

\author{
A. Dorn, ${ }^{1}$ A. Kheifets, ${ }^{2}$ C. D. Schröter, ${ }^{1}$ B. Najjari, ${ }^{1}$ C. Höhr, ${ }^{1}$ R. Moshammer, ${ }^{1}$ and J. Ullrich ${ }^{1,3}$ \\ ${ }^{1}$ Universität Freiburg, Hermann-Herder-Strasse 3, 79104 Freiburg, Germany \\ ${ }^{2}$ Research School of Physical Sciences and Engineering, Australian National University, Canberra, ACT 0200, Australia \\ ${ }^{3}$ Max-Planck-Institut für Kernphysik, Saupfercheckweg 1, 69117 Heidelberg, Germany
}

(Received 14 November 2000)

\begin{abstract}
The dynamics of He double ionization by $2 \mathrm{keV}$ electron impact is studied experimentally for a momentum transfer of 0.6 a.u. at excess energies of 10 and $40 \mathrm{eV}$. Complete sets of fivefold differential cross sections are presented for all electron emission angles in coplanar geometry. Contributions beyond the first Born approximation are identified comparing experimental data with first order convergent close-coupling calculations which are in considerably better agreement with the present experiment than with the earlier measurement of Kheifets et al. [J. Phys. B 32, 5047 (1999)].
\end{abstract}

PACS numbers: $34.80 . \mathrm{Dp}$

A full understanding of the correlated fragmentation dynamics of an atomic system upon impact of a charged particle or a photon is one of the central aims of atomic collision physics. While for the most basic three-body breakup processes such as electron impact single ionization of hydrogen or photo double ionization (PDI) of helium a rather profound understanding is emerging (see [1-3] and numerous references therein), the transition to true many-body systems still remains a challenge, both experimentally and theoretically. With the availability of new experimental multicoincidence techniques, we now have the opportunity to obtain complete momentum space pictures of complex many-body processes such as double ionization of helium by electron impact, a so-called $(e, 3 e)$ reaction leading to four unbound particles $[4,5]$.

On the theoretical side, fairly sophisticated models have been employed [6-8] to describe the first $(e, 3 e)$ experiment on helium [9]. Because of a large disparity in the energies of the fast projectile $(5.6 \mathrm{keV})$ and the slow ejected electrons $(10$ or $4 \mathrm{eV})$, it was natural to limit the projectiletarget interaction to the first order while treating the interaction of the two slow ejected electrons nonperturbatively [6,7]. However, these first Born calculations produced confusing results. Agreement with the experiment was poor, both in magnitude and in shape, especially at the lowest $4 \mathrm{eV}$ ejected electron energy. Some of the experimental features, such as strong peaks in the cross section for small relative electron emission angles, could not be reproduced at all. Agreement with the experiment seemed to improve when a full four-body model was employed [8]. In the meantime, the authors of Ref. [7] did not rule out the possibility that some "hidden" artifacts had actually affected the experiment. Therefore, the matter remains controversial, and clarifying experimental and theoretical studies are highly desirable.

With the aim to resolve this controversy, we present in this Letter the data from a kinematically complete experiment for $2 \mathrm{keV}$ electron impact on helium in which, for the first time, a large part of the 12-dimensional, fourparticle final-state momentum space is mapped with sufficient statistics to extract fully differential cross sections. Thus, theory can be tested for kinematics ranging from the optical limit to high momentum transfer up to 5 a.u. and over a large range of relative emission angles as well as energy partitions between the two ejected electrons. Similarly to the previous experiments [7,9] performed very close to the optical limit, we select here a relatively small momentum transfer of $|\mathbf{q}|=0.6$ a.u., equal energies of the ejected electrons of $E_{b}=E_{c}=5$ and $20 \mathrm{eV}$, and the coplanar geometry where the ejected electrons are emitted within the scattering plane of the projectile. We compare these data with calculations performed within the same first Born model as employed in [7]. The slow ejected electron interaction was treated nonperturbatively using the convergent close coupling (CCC) method. The method is known to yield very reliable quantitative results for the related PDI process when the two electrons are ejected from the helium atom by photon impact $[10,11]$.

The present experiment is performed applying a novel multielectron-recoil-ion coincidence technique which has been demonstrated to enable $(e, 3 e)$ experiments on helium [4]. Details of the working principle and the data analysis are described elsewhere [5,12]. In short, a supersonic jet provided a well localized $(2 \mathrm{~mm}$ diam $)$ and dense $\left(10^{11}\right.$ atoms $\left./ \mathrm{cm}^{3}\right)$ helium target at the intersection point with a pulsed electron beam. Ions and slow electrons produced in ionizing collisions are extracted to opposite directions by means of static electric and magnetic fields and detected by two position sensitive multichannel plate detectors. From the measured positions and times of flight, the momentum vectors of two slow electrons $\mathbf{k}_{b}$ and $\mathbf{k}_{c}\left(E_{b, c}<30 \mathrm{eV}\right)$ and the momentum vector of the recoiling ion $\mathbf{k}_{\mathrm{He}}{ }^{++}$emitted in an $(e, 3 e)$ reaction are determined. The kinematics of the fast scattered electron as well as the momentum q transferred by the scattered projectile follow from momentum conservation: 


$$
\mathbf{k}_{0}-\mathbf{k}_{a}=\mathbf{q}=\mathbf{k}_{b}+\mathbf{k}_{c}+\mathbf{k}_{\mathrm{He}}^{++} .
$$

Here, $\mathbf{k}_{0}$ and $\mathbf{k}_{a}$ are the momenta of the incoming and the scattered projectile, respectively.

The electron detector is equipped with a fast delay-line readout and a multihit time-to-digital converter. Therefore, position as well as arrival time of the second electron emitted in a double ionization event are determined if the flight-time difference between two electrons exceeds $15 \mathrm{~ns}$. Whereas the complete final-state momentum space is mapped for all ions with $\mathbf{k}_{\mathrm{He}}{ }^{++}<5$ a.u., this detector dead time results in a small loss of the total momentum space for the second electron hitting the detector.

In Fig. 1(a), the fivefold differential cross section (FDCS) is given as a function of the ejected electrons emission angles $\theta_{b}$ and $\theta_{c}$ with respect to the forward projectile beam direction. The momentum transfer is $|\mathbf{q}|=0.6 \pm 0.2$ a.u.; the energies of the ejected electrons are $E_{b}=E_{c}=5 \pm 2.5 \mathrm{eV}$. We have chosen a density plot representation to visualize a large range of angles $\theta_{b}$ and $\theta_{c}$ being particularly well suited to study the overall structure of the cross section and to reveal symmetries as well as nodal lines. The angular range which is not affected by reduced detection efficiency due to the electron detectors dead time is inside the circular solid lines in the diagrams.

The cross section pattern consists of a four peak structure. Both peaks in the upper left are equivalent to the peaks in the lower right of the diagram (marked $A$ and $B$ ) since, for symmetric energy sharing $E_{b}=E_{c}$, both ejected electrons are interchangeable.

In Fig. 1 we also present the theoretical CCC result [1(b)] as well as the corresponding photo double ionization cross section [1(c)] obtained by using the phenomenological parametrization from Huetz et al. [13], which is commonly accepted to yield good agreement with experimental data. This parametrization nicely reveals the origin of the observed structures in factorizing the cross section in an angular part arising from photoabsorption and a correlation part describing the electron-electron repulsion in the final state [3]. For equal energy sharing, the angular distribution of the photoelectrons is given by

$$
\sigma_{\mathrm{PDI}}=\left(\cos \theta_{b \mathbf{E}}+\cos \theta_{c \mathbf{E}}\right)^{2} \cdot C(b, c) .
$$

Here, $\theta_{\mathbf{b E}}$ and $\theta_{\mathbf{c E}}$ are the emission angles relative to the electric field vector $\mathbf{E}$. The angular factor mirrors the ${ }^{1} P^{0}$ symmetry of the final state and, hence, it gives rise to zeros in the cross section arising from dipole selection rules: There is a first node (i) for back to back emission of both ejected electrons $\left|\theta_{b}-\theta_{c}\right|=180^{\circ}$ [dashed lines in Figs. 1(a)-1(c)] and a second node (ii) if the sum momentum of the ejected electrons is perpendicular to the electric field vector $\left(\mathbf{k}_{b}+\mathbf{k}_{c}\right) \perp \mathbf{E}$ or, equivalently, $\theta_{\mathbf{b E}}+\theta_{\mathbf{c E}}=180^{\circ}$ (dotted lines in Fig. 1). The correlation factor $C(b, c)$ is zero for parallel emission $\theta_{b}=\theta_{c}$ and gives rise to a broad minimum of the cross section in the vicinity of small relative angles. Since electron a)

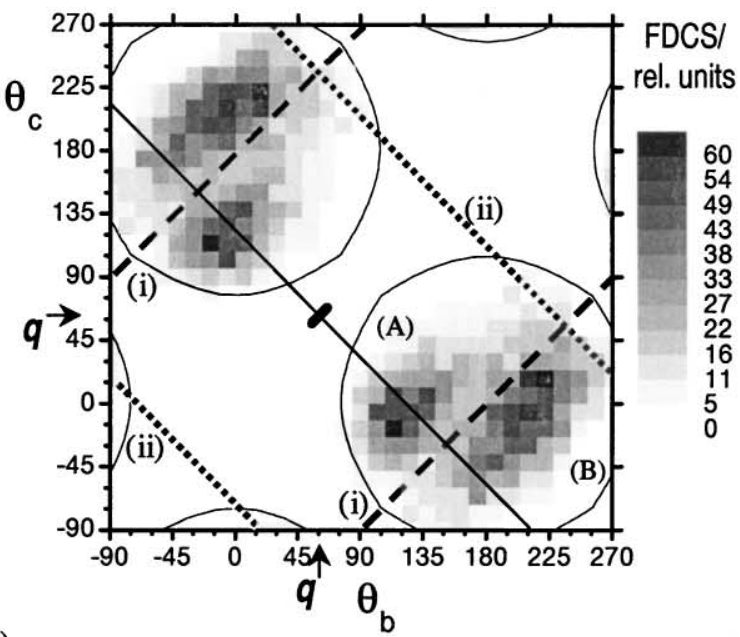

b)

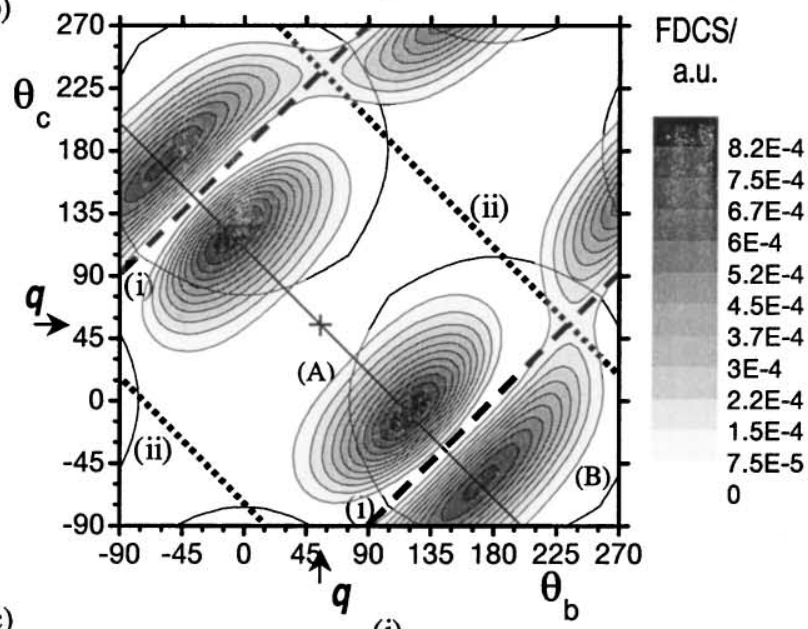

c)

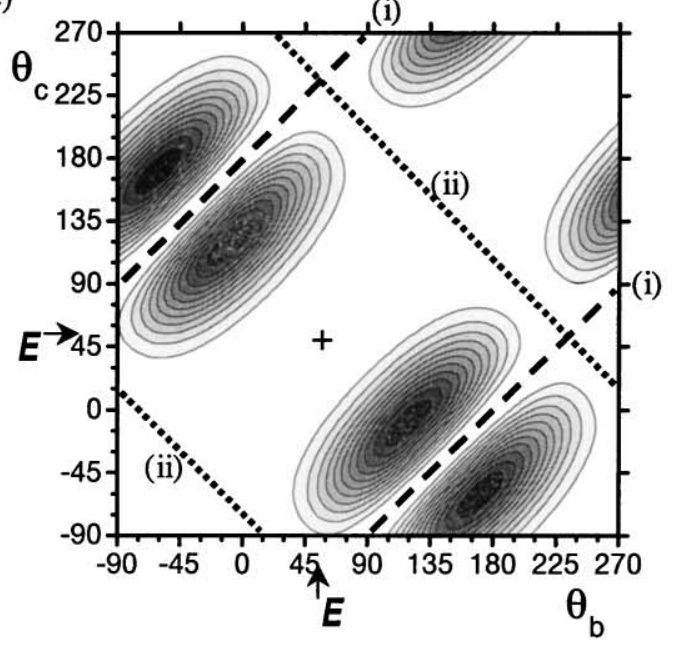

FIG. 1. Fivefold differential cross section in coplanar scattering geometry as a function of the ejected electron emission angles $\theta_{b}$ and $\theta_{c}$ relative to the primary beam forward direction. For all diagrams, the ejected electron energies are $E_{b, c}=5 \mathrm{eV}$. (a) Experimental cross section for $E_{0}=2 \mathrm{keV},|\mathbf{q}|=0.6$ a.u. The direction of the momentum transfer $\mathbf{q}$ is marked by arrows in the diagram. The angular ranges which are not affected by the detector dead time are encircled by solid lines. (b) CCC calculation for $|\mathbf{q}|=0.5$ a.u. (c) Cross section for photo double ionization obtained with the parametrization from Huetz et al. (see text). The electric field vector $\mathbf{E}$ has been oriented to coincide with the corresponding quantization axis $\mathbf{q}$ for the $(e, 3 e)$ process in (b) $\left(\theta_{\mathbf{E}}=\theta_{\mathbf{q}} \approx 55^{\circ}\right)$. 
repulsion acts similarly for the $(e, 3 e)$ process, a large part of the experimentally nonaccessible angular range, for emission of both electrons into the same direction $\theta_{b} \approx \theta_{c}$ at equal energies, is not of major importance as the cross section is close to zero in this region. A further property of the PDI cross section is the reflection symmetry with respect to a plane perpendicular to the electric field axis since both directions along the axis are equivalent and indistinguishable. Therefore the cross section shows reflection symmetry with respect to the lines (i) and (ii). Examining the experimental and theoretical $(e, 3 e)$ cross sections, it immediately becomes obvious that both are governed by the same nodal lines showing close similarities to PDI, despite the fairly large momentum transfer of $|\mathbf{q}|=0.6$ a.u. This has been discussed previously for conditions even closer to the optical limit by Lahmam-Bennani et al. [6]. The experimental uncertainty in the amount of the momentum transfer gives rise to an uncertainty in the direction of the $\mathbf{q}$ indicated by the length of the thick solid line in Fig. 1(b) which marks angles where both electrons are emitted parallel to $\mathbf{q}$. Therefore the peak widths should be smeared out accordingly in this direction. The experimental uncertainty in the electron energies $\mathbf{E}_{b, c}$ can cause the observed partial filling of the node for back to back emission since the corresponding selection rule is strict only for symmetric energy sharing between both electrons.

The remaining differences compared to the PDI cross section can be classified as being, first, due to nondipole contributions and, second, due to higher order interactions with the projectile. First, contributions beyond the dipole approximation are the result of momentum transfers $|\mathbf{q}|$ larger than the minimum transfer for photoabsorption $\left|\mathbf{q}_{\gamma}\right|=E_{\gamma} / c$ and, consequently, do not obey the dipole selection rules. As a result, the cross section in the CCC calculation is finite for back to back emission with one electron going into the momentum transfer direction, i.e., a configuration which is strictly forbidden by both dipole selection rules (i) and (ii). As a further consequence, any $q>q_{\gamma}$ breaks the reflection symmetry of the cross section with respect to a plane perpendicular to the quantization axis since the direction along $\mathbf{q}$ now is distinguishable from the one opposite to q. Thus, in Figs. 1(a) and 1(b), the cross section maximum $(A)$ is stronger and less elongated compared to the maximum $(B)$ for which both electrons enclose angles larger than $90^{\circ}$ with $\mathbf{q}$. These features become even more pronounced if the energies of the ejected electrons are higher. For $E_{b}=E_{c}=20 \mathrm{eV}$ [Figs. 2(a) and 2(b)] peak $(A)$ becomes the dominant structure in the cross section which can be understood as follows: Dipole transitions dominate in the transition matrix element not only in the limit of small momentum transfer, where the transition operator reduces to the dipole operator, but also if the energies of the ejected electrons are small. With decreasing energies $E_{b, c}$, the low angular momentum terms in a partial wave expansion of the two-electron final-state wave function dominate more and more at small distances from the ion, i.e., in the volume which largely determines a)
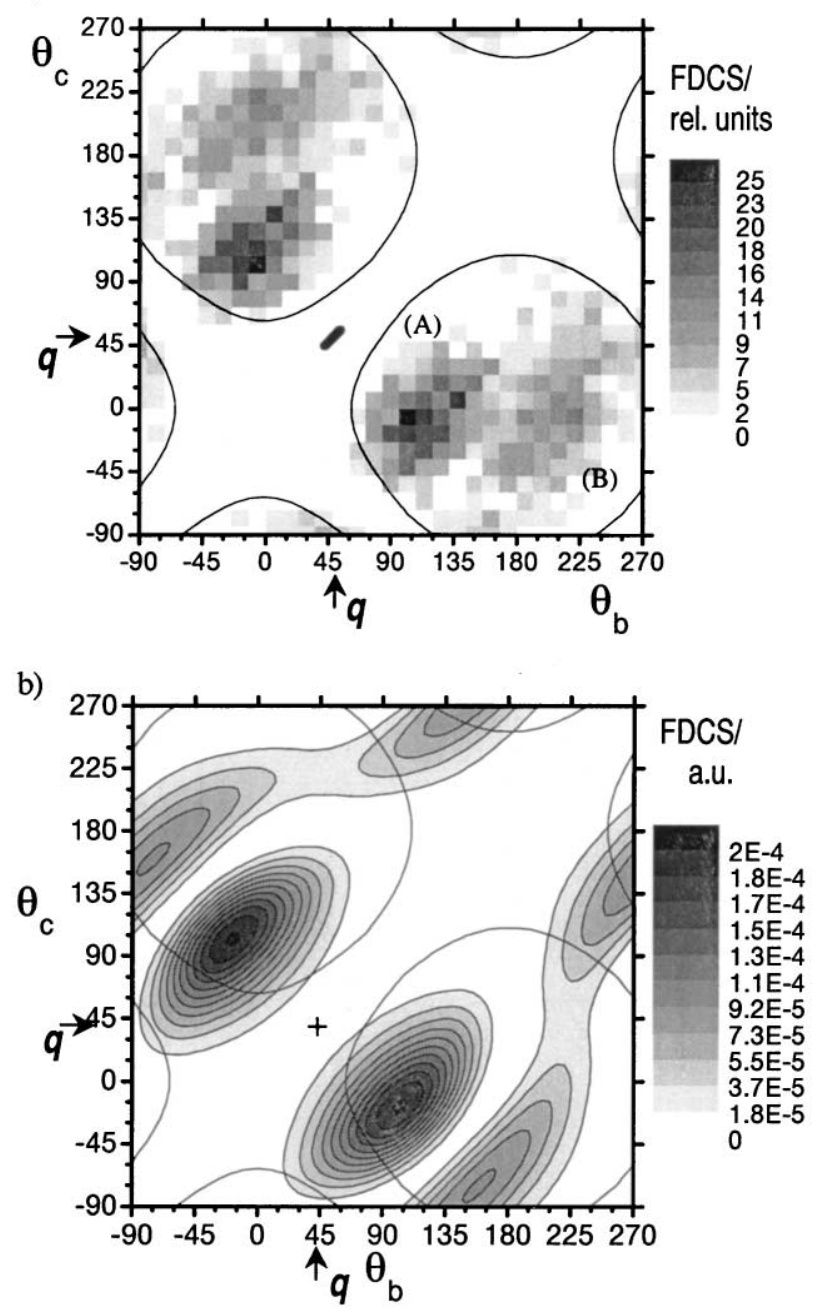

FIG. 2. (a) Experimental FDCS for $E_{b, c}=20 \pm 4 \mathrm{eV}$ and $|\mathbf{q}|=0.6$ a.u. (b) CCC calculation for $E_{b, c}=20 \mathrm{eV}$ and $|\mathbf{q}|=$ 0.5 a.u.

the transition matrix element. Therefore, dipole transitions are emphasized for $E_{b, c}=5 \mathrm{eV}$ despite the relatively large momentum transfer. For $E_{b, c}=20 \mathrm{eV}$, peak $(A)$ is dominant since for the corresponding emission angles so-called Bethe kinematics is closely fulfilled. Here, the sum momentum of the ejected electrons is close to the momentum transfer $\mathbf{k}_{b}+\mathbf{k}_{c} \approx \mathbf{q}$ both in magnitude and direction, i.e., the ion momentum is small. This condition has been shown by Berakdar and Klar [14] to be the preferred collision kinematics for the $(e, 3 e)$ process. Correspondingly, to the nomenclature used for the $(e, 2 e)$ cross sections, we name maximum $(A)$ the "binary peak" and maximum $(B)$ the "recoil peak" since, for this maximum, the electrons sum momentum is directed opposite $\mathbf{q}$ and the residual ion carries considerable momentum.

In all features discussed thus far, experiment and the CCC theory agree remarkably well. The most striking remaining disagreement of experiment and CCC theory, namely, the position of peak $(B)$, can be assigned to higher order projectile-target interactions by the following 
symmetry arguments: A collision process of the first order reveals axial symmetry of the cross section with respect to the momentum transfer direction. Thus, the theoretical cross sections [Figs. 1(b) and 2(b)], which are obtained in first Born approximation for the projectiletarget interaction, are invariant for inversion at the point where both electron's emission angles are equal to the angle of the momentum transfer or, equivalently, for reflection of both momentum vectors at the direction of q. Taking into account the symmetry with respect to the line $\theta_{b}=\theta_{c}$ mentioned previously, it follows that there is also reflection symmetry with respect to the straight solid line in Fig. 1(b) corresponding to electron emission into equal but opposite angles with respect to the momentum transfer direction: $\left(\theta_{b}+\theta_{c}\right) / 2=\theta_{q}$. While in the experimental data this symmetry is closely fulfilled for the binary peak $(A)$, the recoil peak $(B)$ shows a distinct shift to larger angles for both electrons. Therefore, higher order projectile-target interactions are of importance and explain the failure of first order theories for certain parts of the final-state momentum space. For the higher energies of the ejected electrons, the recoil peak $(B)$ decreases strongly in magnitude becoming very weak for $E_{b, c}=20 \mathrm{eV}$ (see Fig. 2). Since peak (B) is the only feature showing signatures of higher order projectile-target interaction, we can clearly prove experimentally previous assumptions made by Kheifets et al. [7], namely, that second order effects in the projectile-target interaction are more important for smaller energies of the ejected electrons $E_{b, c}$.

Finally, the cross section for $E_{b, c}=5 \mathrm{eV}$ as function of the mutual angle $\theta_{b c}=\left(\theta_{b}-\theta_{c}\right)$ integrated over the whole range of the individual angles $\theta_{b}$ and $\theta_{c}$ is presented in Fig. 3. The agreement with theory, which in this case is integrated over the experimental uncertainty of $E_{b, c}$, is quite satisfactory, in particular, if the reduced accessible angular range of the experiment is taken into account (full line in the figure). The width of the cross section minimum for small mutual angles is consistent with observations from PDI at similar excess energies [15]. Thus, within the angular range accessible in the present experiment, we cannot confirm the somewhat surprising experimental observations from Kheifets et al. [7], who reported a four-lobe structure for $E_{b, c}=4 \mathrm{eV}$ with two strong maxima at relative angles of $\theta_{b c}=60^{\circ}$ and $300^{\circ}$. It must be emphasized that these extra lobes where observed for both electrons going to small forward angles, a configuration which is not part of the present accessible angular range [see Fig. 1(a)]. On the other hand, we can confirm a filling of the node at $\theta_{b c}=180^{\circ}$ which cannot be attributed to be due only to instrumental angular resolution but also indicates nonfirst Born processes.

In conclusion, the present experimental data allow for a large scale view of the final-state momentum space and, therefore, enables the identification of general symmetries and structures of the cross section. In contrast to earlier studies, remarkably good agreement with the CCC cal-

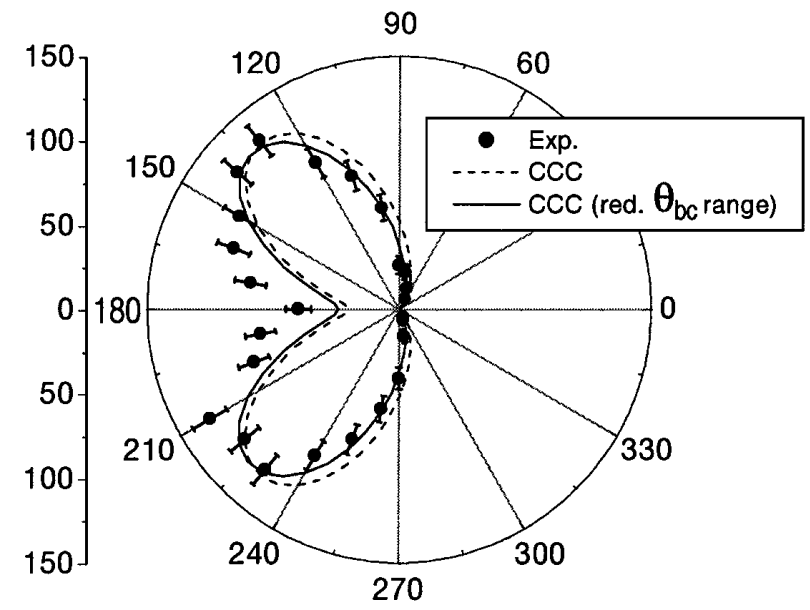

FIG. 3. Cross section as a function of the mutual angle $\theta_{b c}=$ $\left|\theta_{b}-\theta_{c}\right|$ integrated over all values of the individual $\theta_{b}$ and $\theta_{c}$ for $E_{b, c}=5 \mathrm{eV}$. The CCC calculation for the full angular range and for the angular range accessible experimentally is given as a dashed line and a continuous line, respectively.

culations is found concerning all features in the shape of the cross sections which can be described within a first order approach, i.e., relative peak heights and widths as well as the position of nodal lines for both cases $E_{b, c}=5$ and $20 \mathrm{eV}$. We also observe a break of symmetry of the cross section with respect to the momentum transfer direction which is a clear signature of higher order interactions which are not included in theory. For the partially integrated cross section as a function of the mutual angle $\theta_{b c}$, good agreement is obtained which is in contrast to recent experiments closer to the optical limit.

Support from the Deutsche Forschungsgemeinschaft within the SFB 276, project B7, and within the Leibniz program is gratefully acknowledged.

[1] T. N. Rescigno et al., Science 286, 2474 (1999).

[2] C. T. Whelan, Science 286, 2457 (1999).

[3] J. S. Briggs and V. Schmidt, J. Phys. B 33, R1 (2000).

[4] A. Dorn et al., Phys. Rev. Lett. 82, 2496 (1999).

[5] R. Moshammer et al., Nucl. Instrum. Methods Phys. Res., Sect. B 108, 425 (1996); H. Kollmus et al., Nucl. Instrum. Methods Phys. Res., Sect. B 124, 377 (1997).

[6] A. Lahmam-Bennani, I. Taouil, A. Duguet, M. Lecas, L. Avaldi, and J. Berakdar, Phys. Rev. A 59, 3548 (1999).

[7] A. Kheifets, I. Bray, A. Lahmam-Bennani, A. Duguet, and I. Taouil, J. Phys. B 32, 5047 (1999).

[8] J. Berakdar, Phys. Rev. Lett. 85, 4036 (2000).

[9] I. Taouil, A. Lahmam-Bennani, A. Duguet, M. Lecas, and L. Avaldi, Phys. Rev. Lett. 81, 4600 (1998).

[10] A. S. Kheifets and I. Bray, J. Phys. B 31, 5149 (1998).

[11] A. S. Kheifets and I. Bray, Phys. Rev. Lett. 81, 4588 (1998).

[12] J. Ullrich et al., J. Phys. B 30, 2917 (1997).

[13] A. Huetz et al., J. Phys. B 24, 1917 (1991).

[14] J. Berakdar and H. Klar, J. Phys. B 26, 4219 (1993).

[15] O. Schwarzkopf, B. Krässig, and V. Schmidt, J. Phys. (Paris), Colloq. 3, C6-169 (1993). 